EOMmUn Communication et organisation

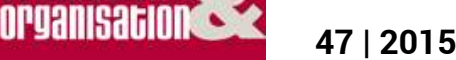

Recherches émergentes en communication des organisations

\title{
Étude exploratoire sur la place des rôles dans l'analyse des pratiques informationnelles
}

Exploratory study on the place of roles in the analysis of informational practices

\section{Anna Lezon Rivière}

\section{OpenEdition}

\section{Journals}

Édition électronique

URL : http://journals.openedition.org/communicationorganisation/4937

DOI : 10.4000/communicationorganisation.4937

ISSN : 1775-3546

Éditeur

Presses universitaires de Bordeaux

Édition imprimée

Date de publication : 1 juin 2015

Pagination : 161-178

ISSN : $1168-5549$

Référence électronique

Anna Lezon Rivière, «Étude exploratoire sur la place des rôles dans l'analyse des pratiques informationnelles », Communication et organisation [En ligne], 47 | 2015, mis en ligne le 01 juin 2018, consulté le 03 mai 2019. URL : http://journals.openedition.org/communicationorganisation/4937 ; DOI : 10.4000/communicationorganisation.4937 


\title{
Étude exploratoire sur la place des rôles dans l'analyse des pratiques informationnelles
}

\author{
Anno Lezon Rivière'
}

\section{Introduction}

Cet article est le résultat d'une étude sur les pratiques informationnelles, menée auprès des chefs militaires exerçant leurs fonctions dans les étatsmajors centraux (Lezon Rivière 2013). Nous présentons ici les premiers résultats exploratoires sur la place des rôles dans l'analyse des pratiques informationnelles. Tout d'abord, nous interrogeons le concept de rôle pour mieux cerner son influence sur la manière dont l'ensemble des dispositifs, des sources, des compétences cognitives sont utilisés dans les différentes situations de production, de recherche, d'organisation et de diffusion de l'information (Ihadjadene \& Chaudiron 2008 : 23). Notre propos dans cet article n'est pas d'apporter les réponses exhaustives à la problématique de la place des rôles dans l'analyse des pratiques informationnelles, mais de contribuer à cette réflexion en s'appuyant sur les résultats de l'étude empirique menée dans le domaine militaire. Notre étude empirique avait pour objectif premier d'explorer les pratiques informationnelles des chefs militaires en s'appuyant sur la méthodologie communicationnelle de construction de sens (sense-making methodology) de Dervin. Lors de nos entrevues, sans que nous formulions explicitement la question, les chefs militaires ont décrit leurs rôles et leurs responsabilités, nous fournissant ainsi la matière pour mieux cerner leurs rôles et la compréhension qu'ils en ont. Ces résultats intermédiaires contribuent à mieux saisir les liens entre le rôle et les pratiques informationnelles des chefs militaires.

\footnotetext{
1 Dr. Anna Lezon Rivière est maître de conférences et chercheure à l'Université de Paris 8, laboratoire Paragraphe, membre de l'équipe Index. Professionnelle en gestion de l'information durant douze ans (audits des pratiques informationnelles, management du centre documentaire, conception et mise en ouvre des systèmes info-documentaires), ses recherches universitaires sont étroitement liées à son parcours professionnel. Ses travaux portent actuellement sur la gestion stratégique de l'information, les pratiques informationnelles et la construction de sens (sensemaking) ; annalezon@gmail.com
} 


\section{Concept de " rôles" : théories et typologies}

Biddle (1986) et Cambell (1999) recensent cinq perspectives à travers lesquelles la théorie des rôles est abordée : fonctionnaliste, interactionniste symbolique, structuraliste, organisationnelle et cognitiviste (Glick $2011: 25$ ), (Louchart 2012 : 64). Dans la perspective fonctionnaliste le rôle répond à des normes prédéfinies par une fonction donnée. Le structuralisme assigne le rôle à une position sociale dans une structure donnée. Selon le paradigme interactionniste le rôle se construit dans l'interaction. Dans l'approche cognitiviste le rôle est examiné au regard des attentes qui émergent et sont formulées à son égard. En effet, « les positions sociales sont des contraintes aux comportements des individus car elles induisent une pression due aux attentes " (Calvo-Ryba 2004). La perspective organisationnelle mentionnée dans la littérature anglo-saxonne englobe les sciences de gestion et donc les travaux de Mintzberg, notamment.

Glick (Glick 2011) indique trois points qui seraient communs aux courants de pensée au sein de cette théorie : 1) elle étudie les individus au sein d'un cadre spécifique : une organisation, 2) ces individus présentant des comportements spécifiques : les rôles, 3 ) ces comportements (les rôles) sont conditionnés par les attentes qui leur sont exprimées. La situation où ces attentes ne sont pas remplies crée « un conflit des rôles » (Glick 2011 : 27). Ces attentes face au rôle qui devrait être joué au sein de l'organisation peuvent manquer de clarté, note Maruca (2000) dans son étude sur le rôle des directeurs des systèmes d'information (CIO - chief information officer) (Louchart 2012 : 223).

Pour conduire l'étude empirique sur la dynamique des cadres supérieurs, Calvo-Ryba définit le rôle de la façon suivante : " le rôle est un ensemble de comportements observables attachés à une position sociale aux normes structurantes et à un construit créatif issu de l'interaction »(Calvo-Ryba, 2004). La définition proposée par cet auteur est issue du rapprochement des paradigmes fonctionnaliste, structuraliste et interactionniste. L'auteur précise que le rôle n'est pas un processus de pensée, mais il est un acte observable. Le rôle est ainsi considéré comme un "épisode observable » de l'action du manageur. "Étudier les rôles des dirigeants, c'est alors vouloir dépeindre une réalité d'un acteur social particulier dont chaque action s'inscrit dans un projet organisationnel précis. » (Calvo-Ryba 2004). Aussi, le rôle est un outil conceptuel qui peut englober une tâche ou une fonction du fait de sa dimension observable.

C'est sans doute l'œuvre de Mintzberg qui est la plus discutée. Sa taxonomie des rôles est largement reprise et citée par des chercheurs, particulièrement en sciences de gestion. Sur la base de ses travaux empiriques, Mintzberg (Mintzberg 2006) identifie dix grands rôles des cadres. Ces rôles sont réunis au sein de trois classes : les rôles interpersonnels, les rôles liées à l'information et les rôles décisionnels. Les rôles interpersonnels sont : symbole (chef symbolique), leader (chef qui motive, qui affecte les personnels), agent de 
liaison (création et entretien des réseaux de contacts externes). La seconde classe des rôles liés à l'information englobe : le rôle d'observateur actif (centre nerveux de l'information externe et interne : recherche et reçoit l'information pour développer « une compréhension approfondie de l'organisation et de l'environnement ", le rôle de diffuseur (transmet les informations reçues, externes et internes), le porte-parole (transmet l'information vers l'extérieur, expert de l'activité de l'organisation). Les rôles décisionnels sont les suivants : entrepreneur (recherche d'opportunités, à l'initiative des changements), le régulateur (responsable des actions correctives), répartiteur des ressources (prise ou approbation des décisions importantes) et enfin, le rôle de négociateur, lors des négociations importantes de l'organisation (Mintzberg 2006 : 104).

Selon Mintzberg et d'autres auteurs, le rôle de leader est considéré comme prépondérant concernant les dirigeants. L'apport récent des théories de leadership suggère que le rôle de leader est un rôle évolutif et se joue non seulement au sein de l'organisation, mais également à l'échelle mondiale et en tant que citoyen (Antonioni 2003; Matsumura \& Shin 2005) in (Glick 2011 : 32). Les études de Mintzberg conduites dans les années 1960 et 1970 ne font pas ressortir cet aspect de ce rôle.

Mintzberg formule quelques caractéristiques du travail de manageur et, également, en lien avec l'information. La grande part du travail d'un cadre est non programmée. Toutefois, dans la partie du travail régulier figure la transmission de l'information. Le cadre est « le point focal du flux d'information » dans son organisation. L'information lui arrive principalement sous forme verbale. Il a une forte préférence pour la communication verbale. Ainsi, « la production d'un cadre peut être mesurée essentiellement en terme d'information transmise verbalement » et les « contacts sont l'essence même de son travail " (Mintzberg 2006 : 56). Il est un nœud informationnel au sein de réseau d'information entre ses contacts externes et ses interlocuteurs internes en filtrant et diffusant l'information. Du fait de la brièveté, de la variété et de la fragmentation de son activité, il est un " manipulateur de l'information » dans un environnement "stimulus-réponse ». Cette étude se base sur l'observation "détaillée " de l'activité des manageurs ainsi que sur l'examen des agendas et l'analyse des dossiers d'activités des dirigeants aux profils divers.

Après la revue de la littérature, Calvo-Ryba (Calvo-Ryba 2005) établit une grille de 104 rôles classés selon la catégorisation des rôles de Mintzberg. C'est ainsi que, par exemple, le méta-rôle diffuseur comporte les rôles : acquérir, traiter et échanger l'information, écrire, recevoir et donner des coups de téléphone, etc. Le rôle de l'observateur actif comprend : scanner l'environnement - veille stratégique ; collecter et traiter l'information, rechercher l'information, s'informer, écouter, etc. On retrouve les rôles liés à la pratique de la communication au sein des méta-rôles comme diffuseur (communiquer la vision stratégique aux personnels), agent de liaison (parler 
avec les personnels, jouer le rôle de canaux de communication), etc. Le métarôle de leader comprend des rôles, tels développer une identité collective, donner un sentiment d'appartenance ; élaborer une confiance mutuelle ; diriger, commander.

Cette étude fait ressortir que la dynamique du dirigeant trouve ses fondements dans les rôles de commandement, mais également de collecte et de traitement de l'information. Il y apparaît également que les rôles liés à l'information sont les rôles les plus fréquemment " joués ». Il en ressort également que « les rôles de l'information sont (...) des moyens d'alimenter ou d'introduire les rôles du leadeurship ». C'est ainsi que "la collecte et le traitement de l'information sont les piliers du leadership »(Calvo-Ryba $2004: 20)$.

Suivant Mintzberg, l'étude de Calvo-Ryba fait appel à la méthodologie d'enquête basée sur l'observation passive. L'exploitation des résultats consiste à isoler et à chronométrer les périodes de temps regroupées par une activité particulière en s'appuyant sur l'analyse interprétativiste des comptes rendus de l'observation et des verbatim des conversations des dirigeants avec d'autres acteurs. Les éléments de contexte pris en compte sont : le secteur d'activité, le chiffre d'affaires et les effectifs. L'étude menée à l'aide de cette méthodologie fournit les résultats quantitatifs de recensement, mais ne semble pas pouvoir répondre à la question « du pourquoi » de ces actions.

Un autre exemple récent de la typologie des rôles est celle réalisée par Glick (Glick 2011) pour le besoin de l'étude empirique statistique sur le rôle des cadres et le temps que les cadres leur consacrent. Après la revue de la littérature, l'auteur indique six catégories de rôles : 1 ) catégorie des rôles informationnels : veilleur (monitor), diffuseur, porte-parole, commandant (donne des ordres), 2) catégorie des rôles interpersonnels 3) catégorie des rôles décisionnels, 4) catégorie des rôles opérationnels, 5) catégorie des rôles stratégiques : coordinateur, innovateur, planificateur (vision setter), stratège, transformateur, celui qui crée et maintien la culture (creator and maintainer of culture), 6) catégorie des rôles diplomatiques : rôle du « lien " (statesperson : assure le lien entre l'organisation et son environnement externe), figure de proue (représente l'organisation), rôle de liaison (interagit avec les pairs et autres acteurs externes pour obtenir des informations, construit les réseaux). Pour établir les catégories, l'auteur a sélectionné 20 études consacrées aux cadres supérieurs, basées sur Mintzberg. Cette étude réalisée aux États-Unis conclut que les cadres consacrent $14,6 \%$ de leur temps aux rôles informationnels, $17,5 \%$ aux rôles interpersonnels, $18,1 \%$ aux rôles décisionnels, $18 \%$ aux rôles opérationnels, $21 \%$ aux rôles stratégiques et enfin 10,8 \% de leur temps aux rôles diplomatiques. Le comparatif avec d'autres études menées semble difficile du fait des typologies difficilement comparables en contenus.

On trouvera dans Johnson (Johnson 2013 :120) une typologie des écoles de pensée et une analyse critique des travaux sur l'activité des manageurs 
au quotidien. Les récentes études se consacrent à éclaircir le lien entre les rôles et l'interculturel, l'influence de la mondialisation sur les pratiques des manageurs et enfin, la place des TIC dans la prise de décision.

Ce rapide tour d'horizon sur le concept du rôle et des notions liées nous enseigne principalement le manque d'homogénéité dans les définitions du concept de rôle selon les courants de pensée ou les domaines des sciences concernées et, ainsi, l'impact que cela peut avoir sur les études menées.

\section{Rôles et pratiques informationnelles}

La place des rôles dans l'analyse des pratiques informationnelles est peu étudiée en sciences de l'information et de la bibliothéconomie. La majorité des travaux se sont focalisés, notamment, sur le besoin d'information et les critères de sélection des sources d'information (de Alwis, Majid \& Chaudhry 2006). Cependant, quelques modèles majeurs de la recherche et de la gestion de l'information s'intéressent aux rôles ou groupes d'acteurs dans l'analyse des pratiques informationnelles. Ainsi, Leckie, Pettigrew et Sylvain (1996) proposent un modèle qui intègre explicitement les " rôles professionnels » dans l'analyse des pratiques informationnelles. Pour ces auteurs, les besoins d'information sont en effet déterminés par les "tâches "2 qui sont ellesmêmes directement déduites des "rôles professionnels ». A chacun de ces rôles correspondent des tâches qui, à la différence des rôles, varient selon les professions (rédaction de rapports, conseil, etc.). Le modèle de Leckie et al. est clairement destiné aux groupes professionnels et l'analyse des pratiques informationnelles y est liée au processus de travail.

Pour sa part, Huvila (Huvila 2008), aborde le « rôle professionnel » (work role) d'une manière plus générale et en lien avec le concept de « travail». Dans son approche, le concept de travail est situationnel, hautement contextualisé et en construction permanente. Il englobe les aspects individuels, sociaux et culturels. L'activité informationnelle est intégrée dans le «travail » dont il constitue une grande partie invisible. L'activité informationnelle, selon ces deux précédents modèles, sont « des activités modelées par l'activité principale et contraintes par les impératifs de celle-ci » (Paganelli 2013).

Pour notre part, nous avons fait appel à l'approche par construction de sens : le modèle de sense-making de Dervin (Dervin 1992), (Dervin 2008) et les travaux en sensemaking de Weick (Weick K. E. 1995), (Weick K. E. 1969), pour conduire notre étude des pratiques informationnelles des chefs militaires.

\section{Méthodologie et échantillon d'acteurs}

Notre projet de recherche visait à explorer les pratiques informationnelles des chefs militaires dans le contexte organisationnel. Pour conduire l'étude empirique nous avons fait appel à la méthodologie de conduite d'entretien par construction de sens : sense-making methodology (SMM) de Dervin 
(Dervin 2008). Ancrée dans le constructivisme, cette méthodologie s'attache à l'analyse des enchaînements des actions dans l'espace-temps, qu'elles soient cognitives ou somatiques. L'entretien débute par le choix d'une situation vécue par l'acteur. Dans le souci de la cohérence de nos données de recherche, nous avons suggéré aux acteurs de choisir une situation où ils seraient confrontés à un manque d'information. L'acteur poursuit le récit des actions entreprises. L'enquêteur se concentre sur le "gap" : la discontinuité propre à la situation (par exemple une lacune dans la compréhension) ainsi que les stratégies utilisées par l'enquêté pour obtenir les résultats, sans les préjuger positifs ou négatifs. Lors de l'entretien, le récit de l'acteur, situé dans l'espace-temps, est abordé à travers des actions : un processus individuel et rétrospectif de construction de sens.

En incluant l'interrogation sur le retour d'expérience (RETEX), en référence aux travaux de construction de sens (sensemaking) de Weick(Weick K.E.1995), nous avons souhaité combler d'éventuels manques en historicité et en lien organisationnel. Nous avons conduit vingt-sept entretiens individuels selon la méthodologie de construction de sens (SMM). Nous avons rencontré neuf officiers généraux et dix-huit officiers ayant plus de vingt années de carrière militaire. Au moment de l'enquête, les chefs militaires interviewés occupaient les postes à haut niveau de responsabilités dans un état-major central. Les trois armées - armées de terre, armée de l'air et marine nationale - étaient représentées dans notre échantillon d'acteurs. Au sein de notre échantillon des chefs militaires nous pouvons distinguer deux corps : officiers généraux et officiers supérieurs.

L'officier général, dans ses fonctions de direction ou de conduite de projets en état-major, agit dans les espaces politique, économique, industriel et de la haute fonction publique. "Les officiers généraux s'inscrivent au sommet de la hiérarchie militaire. Leurs responsabilités et prises de décision, (sont) comparables à celles de la haute fonction publique et des cadres dirigeants du secteur privé » (Ministère de la Défense 2013), mais en " temps de paix » uniquement. L'officier général est, avant tout, un chef militaire-meneur d'hommes, dans l'esprit des valeurs partagées. Nous pourrions décrire un officier supérieur en ces mêmes termes. La différence peut se situer dans le niveau des responsabilités exercées.

\section{Rôles des chefs militaires : analyse des résultats}

$\mathrm{Au}$ sein de l'institution militaire, la doctrine (CICDE, 2011) décrit quatre fonctions opérationnelles. Aussi, "l'action militaire s'articule autour de quatre fonctions opérationnelles englobantes, ou "fonctions-clés »: « commander », « maîtriser l'information », « opérer » et « soutenir » (CICDE 2011 : 73). La fonction « commander» assure la synergie entre les fonctions " opérer ", "soutenir » et " maîtriser l'information ». La fonction " maîtriser l'information » contribue également à alimenter la fonction « commander». 
Les fonctions-clés se déclinent en sous fonctions. Par exemple, la fonction " maîtriser l'information " a quatre "sous-fonctions ": " acquérir la connaissance ", " gérer l'information », « dégrader l'information adverse » et " influencer, coopérer et communiquer». Ces sous fonctions sont subdivisées en activités. C'est ainsi que " gérer l'information » comprend : le management de l'information (la hiérarchisation et la classification de l'information), le partage de l'information (la diffusion et la présentation de l'information), et la sûreté de l'information (la sécurité de l'information). Il serait possible d'envisager les fonctions, les sous-fonctions et les activités comme " rôles " selon la définition de ce concept par Calvo-Ryba.

Les rôles présentés ci-dessous ont été retenus et leurs libellés ont été formulés à partir des propos des acteurs et des textes militaires. Nous ne cherchons pas à établir une typologie exhaustive ni comparative des rôles. Il s'agit pour nous de rendre compte des rôles mentionnés par les acteurs militaires interviewés dans le cadre de l'étude portant sur les pratiques informationnelles des chefs militaires en lien avec la construction de sens (sensemaking). Ces résultats intermédiaires nous amènent à interroger la valeur opératoire du concept de « rôle » dans l'analyse de celles-ci.

\section{Commander}

De façon générale, le philosophe Saint-Sernin stipule que "l'essentiel du commandement (...) consiste moins à donner des ordres, qu'à savoir mettre chacun à sa vraie place " aussi " discerner si une personne pourra tenir un rôle et remplir une fonction est un art » (Saint-Sernin 1979 : 164). Commander comprendrait en soi l'attribution des rôles aux acteurs. Au niveau institutionnel, selon la doctrine militaire, la finalité de la fonction «commander » est principalement « maîtriser la complexité » des engagements contemporains et coordonner l'ensemble des fonctions clés opérationnelles : "opérer », "soutenir » et " maîtriser l'information» (CICDE 2011 : 74).

Dans l'approche militaire canadienne, le commandement repose sur trois piliers : l'autorité, la gestion et le leadership. Chaque pilier est une partie intégrante et souvent interdépendante de commandement. Tandis que la gestion repose sur l'autorité organisationnelle formelle, le leadership est une face « humaine » de commandement (Walker 2007 : 103). Cet élément humain consiste, notamment, à diriger, motiver et inspirer les hommes, particulièrement en temps de crise marqué par la complexité des situations. Il est une composante très individualiste, mais puissante, qui permet aux commandants de tous niveaux de façonner ou modifier l'environnement ou le système avec lesquels évoluent les acteurs et, ainsi, influencer les attitudes, les comportements et les actions des autres (Walker 2007 : 104).

Dans ses recherches en communication militaire, Bryon-Portet (BryonPortet 2008) identifie quatre fonctions du commandement : fonction de décision, fonction d'organisation, fonction de relais vertical-horizontal et la 
fonction de stimulation. Les sociologues militaires évoquent l'incarnation du « charismatique » chef militaire. Cette incarnation du chef est le fruit d'une " appartenance à une structure militaire, l'adhésion à des valeurs et à des buts collectifs » (Martin \& Pajon 2011).

Selon les propos recueillis auprès des acteurs, le chef militaire est " dans l'incarnation du chef » : «le chef a une image ». Le chef militaire c'est celui "vers lequel on se tourne », c'est celui qui « donne le cap ». Le chef militaire assure « le commandement des hommes ». Le commandement « qu'on nous inculque » signifie « tu es responsable de tes hommes » et ces hommes peuvent être amenés à mettre leur vie en jeu. Cette responsabilité s'exerce « les yeux dans les yeux ", alors le chef s'engage dans une "connaissance personnelle » $d e$ "ses hommes »; il établit le « rapport affectif » avec ses subordonnés : « on se sent redevable ".

Le rôle de commandement du chef militaire requiert le dialogue et la capacité à gagner la confiance (CDEF 2010). Il comprend non seulement la dimension symbolique, mais également émotionnelle. Il s'exerce dans la connaissance personnelle des hommes. Ces traits du commandement correspondent aux caractéristiques attribuées par Bennis (Bennis 2009) aux leaders à la différence de celles des manageurs : le manageur se concentre sur les systèmes et la structure tandis que le leader se concentre sur les hommes; le manageur s'appuie sur le contrôle quand le leader inspire la confiance (Bennis 2009 : 41). Ainsi, le rôle « commander » comprendrait les rôles de « symbole » et du « leader » de Mintzberg. Commander peut inclure également le rôle de décision (Bryon-Portet 2008).

\section{Décider}

Décider est inhérent au rôle du chef militaire. L'exercice de ce rôle est, hormis les qualités ayant trait à la personnalité du chef, le résultat de l'apprentissage, un produit de la socialisation au sein de l'institution à travers les expériences vécues, mais aussi les formations suivies : «ce qu'on apprend... c'est préparer les décisions... des décisions dans un contexte de guerre ». Cela demande une « certaine harmonie » dans l'élaboration de la décision et «avoir une confiance absolue dans vos équipes » et « décider vite ». Cette longue pratique de la décision devient ainsi « une habitude », « une culture maison ».

La décision " engageante » d'un chef militaire " c'est un fait d'un homme». Elle est mesurée à la «valeur » de l'homme qui la prend. La décision est prise " après un travail extrêmement collectif », « le décideur seul n'existe pas », le décideur "sait s'entourer». "La façon de traiter les décisions : on essaie de le faire le plus humainement possible.»

Préparer la décision en état-major peut reposer sur « un travail de longue haleine, régulier, répétitif » où d'abord « on sonde, la capacité, la réceptivité de l'organisation » face aux changements à venir. Cela implique de connaître « les vraies postures de chacun et donc de vrais jeux » d'acteurs. Ces propos 
concordent avec Saint-Sernin : «La décision est travail » inséparable du langage et de l'imagination (Saint-Sernin $1979: 6$ ).

Préparer la décision au niveau stratégique de l'organisation nécessite « une interaction avec mes chefs " pour « chercher les orientations dont j'ai besoin à intervalles réguliers dans le raisonnement " pour ensuite " problématiser » et " prioriser». Mintzberg avance que les rôles décisionnels « sont les plus gros utilisateurs d'information " (Maurel, 2006) et cette information est un sens construit dans l'interaction entre les chefs militaires.

De la même façon que le rôle commander, le rôle de décideur du chef militaire est le fruit de l'apprentissage et de l'expérience : du " parcours ». Il se construit dans l'interaction. L'exercice de ce rôle fait partie de la culture de l'institution. Ses caractéristiques sont, notamment, ses aspects collectif et humain. Il sous-tend la confiance. Le contexte premier de ce rôle est celui de la guerre.

\section{Tisser des réseaux}

Tisser des réseaux de travail, d'information, est constitutif des fonctions exercées par les chefs militaires tout le long de leur carrière :

"C'est mon rôle de me trouver des réseaux d'information dans chacun de mes postes. Je suis dans un poste, j'évalue le besoin de l'information et je fais ce qu'il faut pour le trouver. C'est la responsabilité, c'est ma responsabilité ».

« Dans mes différentes postes, j’ai toujours eu à créer les réseaux d'information, à les mettre à ma main en tout cas, à les façonner. Mais, après, ça se fait naturellement. Vous allez rencontrer les gens, il y a une démarche active (...). J'arrive dans ce poste : je suis allé rencontrer mes interlocuteurs, c'est la première étape (...). Je veux insister c'est sur l'initiative personnelle, le rôle de l'individu dans la matérialisation des réseaux, le fait de façonner ses propres réseaux, de les adapter à ce qu'il veut, à sa façon de travailler. »

C'est ainsi que le commandant d'un bateau, d'un porte-avion est « au croisement et au centre » de nombreux réseaux d'information et de personnes :

«Surtout quand on est dans cette fonction (commandant d'un bateau), on est au croisement et au centre de tellement de réseaux d'information et de personnes ; qui vous font converger vers vous des informations, qui sont filtrées d'ailleurs; que d'être pris au dépourvu, c'est finalement très très rare. »

Le rôle de tisser des réseaux du chef militaire est reconnu à part entière. Il est une démarche active et personnelle. La description de ce rôle par les acteurs correspond, en très grande partie, avec les résultats de l'étude auprès des dirigeants des entreprises. Constituer le réseau est aussi l'un des deux grands rôles des dirigeants identifiés par Kotter (Kotter 1982). Les cadres supérieurs y consacrent le temps considérable surtout à l'arrivée dans un poste. 
Ce réseau leur est nécessaire pour construire, élaborer leurs planification et agenda (Kotter 1982 : 67). Il est une importante voire primordiale source d'information. Le réseau est très large et la nature des relations est très diverse et varie en matière de la fréquence et de l'intensité. Cette description du rôle " tisser des réseaux » donnée par Kotter possède plus de correspondances avec nos résultats que le rôle d'agent de liaison de Mintzberg où il s'agit des réseaux de relations externes uniquement.

\section{Veiller les signaux, alerter}

Dans le contexte militaire, en état-major central ou en opération, " la rapidité de l'action est importante ", l'institution a besoin de " garder une sensibilité assez fine sur l'évolution de l'extérieur ». Alors les chefs militaires ont également le rôle de "l'alerteur ». Ainsi, " récupérer sur le terrain les signaux contribue à orienter une stratégie au niveau supérieur ».

«On ne peut pas être (...) dans un mode d'information exclusivement officiel. On ne peut plus nier que l'information qui nous est externe pollue forcément le débat interne. (...). C'est aussi un de mes rôles et j'y suis très vigilant d'où cette veille que j'occupe dès le matin.»

"Récupérer sur le terrain tous les éléments d'information, tous les signaux qui peuvent émerger pour pouvoir analyser tout cela et puis faire remonter vers l'étatmajor les choses qui lui paraissent importantes et qui contribuent finalement à orienter une stratégie au niveau supérieur. »

Nous pourrions rapprocher ce rôle de celui de l'observateur actif de Mintzberg. Calvo-Ryba le complète avec, notamment, le rôle "scanner l'environnement-veille stratégique », le rôle relativement récent au sein des entreprises dans sa description actuelle.

Au sein des armées, veiller les signaux n'est pas un rôle nouveau. Pour citer un exemple, le vice-amiral Willaumez note dans le dictionnaire de la marine : " en armée, escadre ou division il y a, jour et nuit, à bord de chaque bâtiment, un homme chargé de veiller les signaux " (Willaumez 1831 : 566). Cette historicité du rôle, ancrée dans la stratégie et la tactique militaires, lui donne la profondeur temporelle qui ne peut être ignorée dans sa description.

\section{Diffuser l'information}

Assurer la " cohérence de la diffusion » est un des rôles prépondérants des chefs militaires du niveau stratégique de l'organisation. Maîtriser la diffusion est « un acte d'effort collectif » au sein de l'organisation. Toutefois, la diffusion de l'information reste bien un attribut du chef, un acte de commandement : mettre l'information diffusée en perspective, diffuser pour connaître les réactions, ne pas diffuser parce que les acteurs " ne sont pas en état d'avoir l'information tout de suite ", sont autant d'actes des stratégies informationnelles des chefs militaires. 
« Je suis un diffuseur avec filtre on va dire (...) vers le haut et vers le bas aussi. C'està-dire qu'il y a les informations que je ne donne pas parce que j'estime que ce n'est pas la peine que les gens commencent à s'affoler sur telle ou telle chose. Et, il y en a d'autres que je ne donne pas non plus parce que j'attends de savoir si elles vont me remonter naturellement des services subordonnés. Cela permet de savoir comment ça se passe. Cela permet de tester, de temps en temps, si tout fonctionne bien (...). Et puis il y en a d'autres que vous donnez pour savoir comment les gens vont réagir, d'autres que vous donnez parce qu'ils ont besoin de réagir, et parce que vous pensez qu'ils ne sont pas en état d'avoir l'information tout de suite (...). En tout cas il faut avoir en permanence ce souci dans la tête (...). La diffusion est forcément un acte volontaire. »

«Cela fait partie du rôle du commandant de base (la base aérienne) que de s'assurer que les directives se diffusent bien au bon niveau, (...) de mettre en perspective les directives qui tombent, parce que c'est un rôle de commandement. »

Face à l'information externe, la question que se pose le chef est : « jusqu'où dois-je réagir, jusqu'où et comment ? ». Lors de sa diffusion, " est-ce qu'il est important de forcer le trait ou est-ce qu'il est important, au contraire, de donner de la sérénité » à cette information ?

Ce rôle de diffuseur de l'information est largement cité et identifié dans les études sur les dirigeants (Mintzberg 2006), (Calvo-Ryba 2005), (Glick 2011). Chez Mintzberg, le rôle diffuser est un l'un des rôles informationnels et signifie, principalement, transmettre l'information. Calvo-Ryba enrichit ce rôle avec les rôles subséquents : communiquer la vision stratégique ; acquérir, traiter et échanger l'information ou encore informer.

Le rôle de diffuseur du chef militaire relève clairement du commandement. Il serait, malgré son lien évident avec l'information, un rôle interpersonnel de leadership. La diffusion de l'information est également un acte de commandement pour " tester " le fonctionnement des équipes, "voir » les réactions. Cela pose la question de classement des rôles au sein des diverses typologies proposées et, par conséquent, de l'analyse des pratiques informationnelles par le prisme des rôles dissociés.

\section{Donner du sens, construire le sens collectif}

Dans la complexité des situations du domaine militaire, " donner du sens c'est le devoir du chef ». Le chef militaire devrait avoir " une certaine capacité à donner du sens collectif ", " de faciliter une compréhension et un sens commun ", accompagner la compréhension, mais aussi vérifier le sens compris.

«Il y a vraiment cet échange qu'on peut faciliter parce que (...) donner du sens c'est le devoir du chef. (...) Ensuite accompagner, le fait qu’on vérifie qu'il a bien été compris (...) permet aussi d'atténuer ou dire des particularités ou des spécificités. » 
«Mon rôle, surtout dans la partie qui reste en métropole et un peu moins pour la partie déployée sur les théâtres, c'est de m'assurer que tout ce qu'on fait a du sens (...).»

«Face à la complexité de l'interarmées : (...) il faut (...) des gens (avec) une certaine expérience ou une certaine capacité à donner du sens collectif. »

D'autant plus que le sens commun des mots prononcés a tout son poids dans l'exercice du métier des chefs militaires : « Chez nous, plus qu'ailleurs les mots tuent. Parce que, quand vous prononcez un mot, mais que vous n'êtes pas compris (...) ça peut être dramatique. »

Dans ses travaux en construction de sens (sensemaking), Weick (Vidaillet 2003 : 82) prend l'exemple du commandant de bord d'un équipage d'avion pour aborder le leadership ancré dans la construction de sens. Il consacre plus de temps à la mise en place de l'équipe lors de sa constitution. Durant leurs consignes, il dédie plus de temps aux éléments de contexte qu'aux tâches routinières. C'est pourquoi nous considérons « donner, construire le sens collectif » comme rôle de commandement que nous pourrions inclure dans les rôles de leadership au sein des rôles interpersonnels.

\section{Expérience des rôles et pratiques informationnelles}

Les atouts évoqués du chef militaire sont la formation " au commandement», l'expérience "sur laquelle on capitalise » ainsi que l'entraînement : «ce sont les schémas que l'on répète à l'entraînement qui font qu'on peut agir vite, à partir de l'information ». C'est ainsi que « le réflexe » et « l'intuition » sont " travaillés".

«Avant de commander le porte-avion, j'ai commandé trois autres bâtiments, où j'ai vécu les situations plus ou moins faciles, où j'ai fait des erreurs, où j'ai appris à tirer les enseignements de ces erreurs (...) ce qui fait que votre jugement s'affine, vos réactions s'améliorent : c'est ce que j'appelle l'expérience, on n'arrive pas comme ça par hasard. »

"Le système de combat (d'un avion de chasse) est de plus en plus compliqué ; remonte de plus en plus de l'information ; (...) il faut trier l'information et ensuite en fonction des cas étudiés tout le long de la carrière, faire un choix rapide (...). Ce sont les schémas que l'on répète à l'entraînement qui font qu'on peut agir vite, à partir de l'information."

Cet entraînement " tout le long de la carrière " permet d'appliquer les réflexes acquis à d'autres situations, en état-major par exemple : " la vitesse d'analyse des informations ", "la priorisation " s'agissant d'un pilote de chasse :

«Je m'en sers, je pense, dans la priorisation des informations dont j'ai besoin et qui me remontent. Parce qu'une fois j'ai bien priorisé, alors je peux m'en servir rapidement. (...) Et ensuite à réorganiser (...) : quand on est en vol, comme la situation 
n'est pas celle qui était écrite sur le papier, il faut toujours réorganiser, tout le temps (...).»

Cette expérience de la pratique est bien présente dans les textes militaires. Ils précisent que la qualité d'intuition désigne « un lent travail continu de réflexion personnelle » qui accompagne toute la carrière du chef militaire (CDEF, 2010). Il permet d'appréhender et « d'emmagasiner » des exemples de situations et, ainsi, de réagir « très vite » en temps de crise.

Cette expérience nécessaire à l'exercice des rôles est nommée " parcours » :

"Nous ne sommes pas recrutés comme colonel, comme général (...) Nous ne sommes pas recrutés sur un poste. On arrive sur un poste du fait d'un parcours (...) La compétence, c'est-à-dire connaître les subtilités, cela s'apprend. Et cela s'apprend en théorie et puis après cela se pratique, c'est là où arrive la confiance. (...) Il est compétent parce qu'il a eu des examens (...), deux, il a vécu ça donc il sait la nécessité du parcours. »

Aussi, si nous considérons les rôles de la perspective de sensemaking de Weick (Weick K. E. 1995 : 17), leur description tiendra compte de leur ancrage dans la construction réflexive identitaire (réflexion personnelle), de la rétrospective des actions (d'emmagasiner des exemples, parcours) et de leur caractère social (la pratique collective qui instaure la confiance). Nous pouvons également nous référer à Taylor (Taylor, 1991) dans sa description du groupe d'acteurs selon sa formation et sa profession (compétences professionnelles, expertise). Les propos des acteurs confirment l'importance de ces éléments dans l'analyse des pratiques informationnelles.

\section{Discussion et conclusion}

Le contexte militaire possède un certain nombre de particularités. Parmi ces facteurs nous pouvons citer : le passage d'une gestion ordinaire à une gestion de crise (guerre, conflit), la dimension internationale, le facteur politique (Resteigne \& Soeters, 2008). En effet, l'action militaire est dépendante de la décision politique. Ainsi, l'agir militaire n'échappe pas à la complexité politique (Soeters, van Fenema, \& Beeres, 2010). Ces mêmes auteurs indiquent également l'accroissement de la pluralité du travail et du contexte militaire devenu multitâches, multiservices, multinational et multi partenariats. Les chefs militaires agissent dans un environnement dynamique qui se caractérise par de constantes évolutions, liées aux changements des objectifs, des missions et au développement des technologies, notamment. Dans l'analyse de la profession militaire est à éviter l'amalgame que l'on pourrait être tenté de faire entre un chef d'entreprise et un chef militaire.

«Les deux professions peuvent posséder des caractéristiques communes, mais leurs objectifs et leur finalité sont différents. La première est fondée sur deux principes : les résultats nets et la responsabilité limitée ; la responsabilité de l'autre ne connaît 
pas de limites et l'état des profits et des pertes se décline en vies humaines (Horn \& Harris 2002 : 56).»

Notre étude empirique auprès de vingt-sept chefs militaires du niveau stratégique de l'organisation nous a permis d'identifier quelques rôles principaux de ces acteurs militaires. Ces résultats arrivent en complément de notre objectif premier de la recherche qui était d'explorer les pratiques informationnelles de ce groupe d'acteurs. Notre prisme d'étude était la situation de travail dans l'espace-temps où l'acteur a été confronté à un manque de l'information ou une rupture de l'activité de travail. Nous avons privilégié cette approche sous-tendant la construction de sens (Dervin 1992), (Dervin 2008), à l'approche par le questionnement sur les rôles du fait de la richesse des données de recherche qu'elle nous procurait. En effet, cette approche permet d'obtenir des « ensembles » situationnels contextualisés avec les éléments tout aussi individuels qu'organisationnels en lien et à l'origine des pratiques informationnelles.

Les propos des acteurs font ressortir six rôles principaux : commander, décider, tisser des réseaux, veiller les signaux, diffuser l'information et donner du sens-construire le sens collectif. Le rôle nouveau, par rapport aux typologies des auteurs cités, est celui de "donner du sens-construire le sens collectif». Le rôle dont la définition ne correspond pas entièrement à celle donnée par Glick (Glick 2011) est le rôle " commander ». En effet, commander dans le domaine militaire possède une signification bien plus riche que « donner des ordres " ou diriger. En revanche, le rôle qui semble similaire entre les dirigeants des entreprises (Kotter 1982) et les chefs militaires, est le rôle «tisser des réseaux».

Cependant, le comparatif entre les études et les typologies des rôles demeure fragile. Nous avons pu constater que les finalités tout comme les méthodologies utilisées diffèrent selon les domaines scientifiques voire les auteurs. Les définitions elles-mêmes des rôles varient selon le courant de pensée, le champ de recherche ou l'objectif de l'étude empirique. Cet ensemble de facteurs pose nécessairement problème pour aborder la place du rôle dans l'analyse des pratiques informationnelles. Aussi, s'imposent la description rigoureuse de la méthodologie d'enquête des études menées ainsi que du référentiel théorique convoqué pour définir le concept du rôle et de ses typologies.

Leckie et Pettigrew (Leckie \& Pettigrew 1997 : 109) identifient la limite dans les études menées selon l'approche organisationnelle et de sciences de gestion. Linformation y est souvent présentée comme étant liée uniquement à certains postes de direction. Cette approche a tendance à négliger l'importance de l'information dans d'autres rôles du dirigent. Choo et Auster (1993) constatent que "l'information est au cœur de toutes les facettes du travail managérial " (Leckie \& Pettigrew 1997 : 109) C'est pourquoi les recherches en sciences de l'information et en bibliothéconomie s'inscrivent 
dans une perspective plus large. Elles démontrent que l'information est recherchée et utilisée dans tous les multiples rôles professionnels dans une mesure plus ou moins grande. Toutefois, les auteurs estiment que la théorie des rôles a un potentiel considérable pour fournir un cadre conceptuel dans la compréhension de la recherche d'informations des professionnels. Étudier d'autres concepts issus de la théorie des rôles, comme par exemple, le « conflit des rôles " enrichirait la compréhension de la recherche de l'information au sein de l'environnement de travail (Leckie \& Pettigrew 1997 : 109).

Le modèle des pratiques informationnelles centré sur les rôles professionnels de Leckie et al. trouve bien son application auprès des professions où le besoin informationnel est fortement influencé par les tâches liées aux rôles professionnels exercés. Plusieurs études (Landry 2006), (Guclu 2011) ont été menées selon ce modèle, démontrant la pertinence de ce cadre d'analyse dans les contextes professionnels. En revanche, ce cadre d'analyse demeure limité dans l'analyse des pratiques informationnelles des dirigeants et des chefs militaires qui endossent de nombreux rôles au spectre très large d'activités. C'est pourquoi l'analyse seule des pratiques informationnelles par rôles professionnels nous semble insuffisante.

L'étude menée auprès des cadres supérieurs de la santé (Niedźwiedzka 2003) indique que les cadres supérieurs privilégient la médiation d'autres personnes pour accéder à l'information. Les caractéristiques de leur travail comme la surcharge de travail, le manque de temps, la diversité des tâches et la fragmentation de la journée de travail font que les cadres ne disposent pas de temps pour rechercher l'information personnellement, méthodiquement et systématiquement. Les conclusions de cette étude correspondent en partie avec les résultats de notre recherche concernant les chefs militaires. Ces derniers privilégient également le contact humain dans la recherche de l'information.

Notre proposition du cadre d'analyse des pratiques informationnelles des chefs militaires (Lezon Rivière 2013) poursuit l'approche "située " et par construction de sens de l'étude menée. Dans notre modèle, les pratiques informationnelles sont examinées dans le temps (passé, présent, futur), dans les espaces (militaire, politique, social, etc.), à travers la construction de sens individuel (information comme sens construit) et collectif (culture, valeurs, socialisation, retour d'expérience) et selon les variables de la confiance, de la structure et des réseaux. Cette dernière est conceptualisée comme "système des rôles " ancré dans l'approche de sensemaking de Weick (Weick K. E. 1995). Selon les résultats de l'étude, les chefs militaires du niveau stratégique de l'organisation considèrent leurs rôles en relation avec l'ensemble de la structure dont ils relèvent ainsi que de ses interdépendances. 


\section{BIBLIOGRAPHIE}

BENNIS W. G. (2009), On Becoming a Leader. Basic Books.

BRYON-PORTET C. (2008), L'essor de la communication interne dans les armées et ses limites : du commandement au management ? Communication E organisation, 34, p. $154-177$.

CALVO-RYBA J. (2005, juin 6-9), La dynamique du dirigeant au quotidien. Consulté le $1^{\text {er }}$ octobre, 2014, sur AIMS - Association Internationale de Management Stratégique: http://www.strategie-aims.com/

CALVO-RYBA J. (2004), Le travail du dirigeant : vers une analyse en termes de rôle. $13^{\mathrm{e}}$ conférence de l'AIMS. Normandie. Vallée de Seine. 2, 3 et 4 juin.

CDEF. (2010), L'exercice du commandement en opérations pour les chefs tactiques : FT-05. Paris: Ministère de la Défense FR.

CICDE. (2011), Doctrine d'emploi des forces : Doctrine interarmées DIA-01_ $\mathrm{DEF}(2011)$. Centre interarmées de concepts, de doctrines et d'expérimentation. Paris: Ministère de la Défense FR.

DE ALWIS G., MAJID S. \& CHAUDHRY A. S. (2006), Transformation in managers' information seeking behaviour: A review of the literature. Journal of Information Science, 32 (4), p. 362-377.

DERVIN B. (1992), From the mind's eye of the user: the sense-making qualitativequantitative methodology. Dans J. D. Glazier, \& R. R. Powell, Qualitative Research in Information Management. Englewood: Libraries Unlimited, p. 61-84.

DERVIN B. (2008), Interviewing as dialectical practice : sense-making methodology as exemplar. Presented to: audience section International Association for Media and Communication Research (IAMCR), IAMCR 2008 annual meeting. Stokholm, Sweden.

GLICK M. B. (2011), The Role of Chief Executive Officer. Thesis. Fort Collins: Colorado State University.

GUCLU I. (2011), The information-seeking behavior of police officers in turkish national police. Doctoral thesis. University of North Texas.

HORN B. \& HARRIS S. J. (2002), La fonction de général et l'art de l'amirauté : perspectives du leadership militaire canadien. Toronto: Dundurn Press.

HUVILA I. (2008), Work and work roles: A context of tasks. Journal of Documentation, 64, pp. 797-815.

IHADJADENE M. \& CHAUDIRON S. (2008), L'étude des dispositifs d'accès à l'information électronique : approches croisées. Dans F. Papy, Problématiques émergeantes dans les sciences de l'information. Lavoisier.

JOHNSON B. M. (2013), Understanding the work of top managers: A shadowing study of Canadian healthcare CEOs. Doctoral thesis. University of Warwick.

KOTTER J. P. (1982), The General Managers. New York: The Free Press. 
LANDRY C. F. (2006), Work Roles, Tasks, and the Information Behavior of Dentists. Journal of the American Society for Information Science and Technology, 57 (14), pp. 18961908.

LECKIE G. J. \& PETTIGREW K. E. (1997), A General Model of the Information Seeking of Professionals: Role Theory through tne Back Door, In ISIC '96 Proceedings of an international conference on Information seeking in context. London: Taylor Graham Publishing, pp. 99-110.

LEZON RIVIERE A. (2013), Gestion stratégique de l'information et construction de sens : étude des pratiques informationnelles des chefs des militaires. Thèse. Université Paris 8 Vincennes-Saint-Denis.

LOUCHART E. (2012), The Changing Role of the Chief Information Officer: An Inquiry into the Demands, Constraints and Choices of the CIO. Thesis. University of Northampton.

MARTIN C. \& PAJON C. (2011), Max Weber, le charisme routinisé et l'armée de l'air. L'éducation charismatique au sein d'une école d'officiers. Année sociologique, 61, pp. 383-405.

MAUREL D. (2006), Les cadres intermédiaires et l'information : modélisation des comportements informationnels de cadres intermédiaires d'une municipalité en transformation. Thèse de doctorat. Montréal : Université de Montréal.

MINISTĖRE DE LA DÉFENSE (2013), Profil d'un officier général. Consulté le 5 Octobre 2013 sur Ministère de la Défense: http://www.defense.gouv.fr/

MINTZBERG H. (2006), Le manager au quotidien : les dix rôles du cadre. Paris: Eyrolles.

NIEDŹWIEDZKA, B. (2003, October), A proposed general model of information behaviour. Consulté le 21 octobre 2014, sur IR InformationResearch: http://www. informationr.net/ir/9-1/paper164.html

PAGANELLI C. (2013), Une approche info-communicationnelle des activités informationnelles en contexte de travail : acteurs, pratiques et logiques sociales. HDR. Library and information sciences. Université de Grenoble.

RESTEIGNE D. \& SOETERS J. (2008), Managing militarily. Armed Forces E๐ Society, 35 (2), pp. 307-32.

SAINT-SERNIN B. (1979), Le décideur. Paris: Gallimard.

SAVOLAINEN R. (2008), Everyday Information Practices: A Social Phenomenological Perspective. Plymouth: The Scarecrow Press.

SIMONNOT B. (2012), L'accès à l'information : moteurs, dispositifs et médiations. Cachan: Lavoisier.

SOETERS J., VAN FENEMA P. C. \& BEERES R. (2010), Managing Military Organizations: Theory and practice. New York: Routledge.

TAYLOR R. (1991), Information use environments. Dans B. Dervin, \& M. Voigt, Progress in Communication Sciences. Norwood: Ablex Publishing, pp. 217-255.

VIDAILLET B. (2003), Le sens de l'action: KarlE. Weick: sociopsycologie de l'organisation. Paris: Vuibert. 
WALKER R. W. (2007), Institutional leadership in the Canadian forces: Contemporary issues. Kingston, Ontario: Canadian Defence Academy Press.

WEICK K. E. (1995), Sensemaking in Organizations. Sage, California: Thousand Oaks.

WEICK K. E. (1969), The social psychology of organizing. Addison-Wesley : Reading, Mass.

WILLAUMEZ J.-B.-P. (1831), Dictionnaire de la marine avec huit planches.

Résumé: Le tour d'horizon sur le concept du rôle et des notions liées nous enseigne le manque d'homogénéité dans les définitions selon les courants de pensée ou les domaines des sciences concernées. C'est pourquoi notre propos dans cet article n'est pas d'apporter les réponses exhaustives à la problématique de la place des rôles dans l'analyse des pratiques informationnelles, mais de contribuer à cette réflexion en s'appuyant sur les résultats d'études empiriques menées dans le domaine militaire. L'objectif premier était d'explorer les pratiques informationnelles des chefs militaires en s'appuyant sur la méthodologie communicationnelle de construction de sens (sense-making methodology) de Dervin. Lors de nos entrevues, sans que nous formulions explicitement la question, les chefs militaires ont décrit leurs rôles et leurs responsabilités, nous fournissant ainsi la matière pour mieux cerner leurs rôles et la compréhension qu'ils en ont. Nous ne cherchons pas à établir une typologie exhaustive des rôles. Il s'agit ici de résultats intermédiaires permettant de mieux saisir les liens entre le rôle et les pratiques informationnelles des chefs militaires.

Mots-clés : pratiques informationnelles, rôles, chefs militaires, domaine militaire, expérience, construction de sens.

Abstract: The overview on the concept of the role and the concepts related to it, shows the lack of homogeneity in the definitions within the different studies and works on this domain. Therefore, our purpose in this article is not to provide exhaustive answers to the place and importance of roles in the analysis of information practices, but to contribute to this debate, by taking into account the results of the empirical studies done in the military field. The main objective of our empirical study, was to explore information practices of military high commanders, trough the scope of Dervin sense-making Methodology. Without formulating the question explicitly, commanders described their roles and responsibilities and provided us with the material to better understand their roles. We are not seeking to establish an exhaustive typology of roles. This paper is the intermediate result that allows to better understand the links between the role and the information practices of commanders.

Keywords: information practices, role, commanders, military field, experience, sense-making methodology. 